\title{
The Human Microbiome as a Focus of Antibiotic Discovery: Neisseria mucosa Displays Activity Against Neisseria gonorrhoeae
}

\author{
Ellen L. Aho*, Jenie M. Oglet and Anna M. Finck ${ }^{\dagger}$ \\ Department of Biology, Concordia College, Moorhead, MN, United States
}

Neisseria gonorrhoeae infections are a serious global health problem. This organism has developed disturbing levels of antibiotic resistance, resulting in the need for new approaches to prevent and treat gonorrhea. The genus Neisseria also includes several members of the human microbiome that live in close association with an array of microbial partners in a variety of niches. We designed an undergraduate antibiotic discovery project to examine a panel of nonpathogenic Neisseria species for their ability to produce antimicrobial secondary metabolites. Five strains belonging to the N. mucosa species group displayed activity against other Neisseria in delayed antagonism assays; three of these were active against $N$. gonorrhoeae. The antimicrobial compound secreted by N. mucosa NRL 9300 remained active in the presence of catalase, trypsin, and HEPES buffer, and effectively inhibited a DNA uptake mutant of N. gonorrhoeae. Antimicrobial activity was also retained in an ethyl acetate extract of plate grown N. mucosa NRL 9300. These data suggest N. mucosa produces an antimicrobial secondary metabolite that is distinct from previously described antigonococcal agents. This work also serves as a demonstration project that could easily be adapted to studying other members of the human microbiome in undergraduate settings. We offer the perspective that both introductory and more advanced course-based and apprentice-style antibiotic discovery projects focused on the microbiome have the potential to enrich undergraduate curricula and we describe transferrable techniques and strategies to facilitate project design.

This article was submitted to Systems Microbiology, a section of the journal

Frontiers in Microbiology

Received: 29 June 2020 Accepted: 09 November 2020 Published: 03 December 2020

Keywords: human microbiome, antibiotic discovery, undergraduate research, antibiotic resistance, nonpathogenic Neisseria species, Neisseria mucosa, gonorrhea, secondary metabolites

\section{INTRODUCTION}

Citation:

Aho EL, Ogle JM and Finck AM (2020) The Human Microbiome as a Focus of Antibiotic Discovery: Neisseria mucosa Displays Activity Against Neisseria gonorrhoeae.

Front. Microbiol. 11:577762. do: $10.3389 /$ fmicb.2020.577762

Antimicrobial resistance (AMR) in Neisseria gonorrhoeae represents a major public health concern. This human-restricted Gram negative diplococcus is responsible for approximately 87 million cases of gonorrhea per year globally (Rowley et al., 2019). Sexual transmission of N. gonorrhoeae can result in infection of the female and male urogenital tract, rectum, and pharynx. In addition, infected women can transmit the organism during delivery, resulting in neonatal conjunctivitis. Treatment options are generally limited to dual therapy with two antibiotics (ceftriaxone plus azithromycin), and gonococcal strains exhibiting resistance to all recommended antimicrobial 
agents have recently been identified (Eyre et al., 2018; Whiley et al., 2018; Unemo et al., 2019). This pressing global problem has prompted the US Centers for Disease Control and Prevention (2012), World Health Organization, Department of Reproductive Health and Research (2012), and European Centre for Disease Prevention and Control (2019) to develop public health response plans addressing $N$. gonorrhoeae AMR, and new approaches for the prevention and treatment of gonococcal infections are desperately needed.

Neisseria species include $N$. gonorrhoeae, N. meningitidis, which can asymptomatically colonize the human nasopharynx and also cause deadly meningococcal meningitis, several nonpathogenic species that are components of the normal human oropharyngeal microbiome, and nonhuman isolates (Liu et al., 2015). The genus Neisseria is among the ten most abundant genera in the human microbiome (Human Microbiome Project Consortium, 2012), and co-colonization and horizontal gene transfer involving pathogenic and nonpathogenic Neisseria are well documented (e.g., Spratt et al., 1992; Maiden, 1998; Marri et al., 2010; Weyand, 2017). The nonpathogenic Neisseria are of particular interest because natural products produced by members of the human microbiome may represent a source of novel antimicrobial agents (Donia and Fischbach, 2015; Wilson et al., 2017; Milshteyn et al., 2018). For example, the commensal organism Staphylococcus lugdunensis found in the human nasal microbiome produces the secondary metabolite lugdunin, which displays antimicrobial activity against AMR S. aureus (Zipperer et al., 2016). Little is known about production of secondary metabolites by Neisseria, however, some nonpathogenic species have been shown to inhibit pathogens. N. lactamica (Deasy et al., 2015) and N. cinerea (Custodio et al., 2020) inhibit the colonization of $N$. meningitidis in human infection and epithelial cell culture models, respectively, and DNA released by $N$. elongata and multiple other nonpathogenic Neisseria species kills $N$. gonorrhoeae (Kim et al., 2019). In the project described here, we initiated a search for neisserial antimicrobial secondary metabolites by analyzing a panel of 36 nonpathogenic Neisseria strains for activity against other Neisseria, including $N$. gonorrhoeae. We identified five active isolates, all from the N. mucosa species group, and used inhibitor studies and chemical extraction to characterize the activity displayed by N. mucosa strain 9300.

This work was accomplished in a liberal arts college setting utilizing both traditional apprentice-style and coursebased undergraduate research approaches that included both introductory and more advanced students. Projects focusing on AMR and antibiotic discovery have proven to be particularly effective at involving undergraduate students in meaningful research, connecting them to important social issues, and enlivening their study of various aspects of microbiology (e.g., Hoskisson et al., 2015; Smyth, 2017; Dube, 2018; GennéBacon and Bascom-Slack, 2018; Hernandez et al., 2018). Many prior projects focused on searching for antibiotic producing bacteria in environmental samples. For example, Tiny Earth ${ }^{1}$ represents a highly successful initiative in which students

${ }^{1}$ https://tinyearth.wisc.edu/ analyze soil for the presence of bacteria that display inhibitory activity against a panel of target strains, or "safe relatives," closely related to a set of pathogens commonly responsible for nosocomial infections displaying AMR (Hernandez et al., 2018). Here, we offer the perspective the human microbiome represents another ecosystem that is ripe for undergraduate antibiotic discovery projects. We describe tools for screening nonpathogenic members of the microbiome for antimicrobial activity and offer a multiphasic project design for engaging different populations of undergraduate students in traditional and course-based research as a project evolves over time. The strategies, methods, and results described below demonstrate the scientific promise of this approach and offer an undergraduate research model that might be adapted and expanded by others interested in microbiome-based natural products discovery.

\section{MATERIALS AND METHODS}

\section{Bacterial Isolates, Growth Conditions, and Species Confirmation by rpIF Sequence Analysis}

Table 1 lists the nonpathogenic Neisseria strains used in this study. Neisseria were grown on GCB agar (Difco Laboratories) containing Kellogg's supplements I and II (Kellogg et al., 1963) and incubated at $37^{\circ} \mathrm{C}$ with $5 \% \mathrm{CO}_{2}$. Isolate identity for $\mathrm{NRL}$ strains was confirmed by PCR amplification and sequencing of a $413 \mathrm{bp}$ fragment of the $r p l F$ gene followed by analysis using the PubMLST Neisseria database as previously described (Bennett et al., 2014; Jolley et al., 2018). The rplF sequences generated in this study were deposited in GenBank under the accession numbers given in Table 1. Strains were assigned to species groups as proposed by Bennett et al. (2012). The following well-established laboratory strains of $N$. gonorrhoeae were used as targets to test for antibiotic activity: ATCC 19424, ATCC 43070, FA19, F62, MS11, FA1090, and FA6140.

\section{Screening for Antimicrobial Activity}

Delayed antagonism cross-streak assays were used to test 24-h cultures of nonpathogenic Neisseria for their ability to produce antimicrobial substances (Figure 1). A linear streak of each potential producer isolate was made down the center of a GCB agar plate. Plates were incubated for $48 \mathrm{~h}$ and target strains were then streaked at right angles to the line of original growth. Plates were examined after $24 \mathrm{~h}$ of additional incubation for inhibition of target strain growth in the vicinity of the central streak. A strain was considered positive for antimicrobial activity against the target if a clear zone greater than $0.5 \mathrm{~mm}$ was present where the target strain had been inhibited $(n=$ at least three trials per strain for each target).

A confirmatory agar overlay assay was performed on all Neisseria strains that displayed antimicrobial activity in the initial cross streak screening. PBS (Amresco) suspensions containing $10^{9} \mathrm{CFU} / \mathrm{ml}$ of producer strains were prepared from $24 \mathrm{~h}$ plate cultures and $10 \mu \mathrm{l}$ samples of the suspensions were spotted 
TABLE 1 | Inhibitory activity of nonpathogenic Neisseria in delayed antagonism assays.

Target Strains

${ }^{1}$ All species were isolated from human hosts unless otherwise indicated in parentheses.

${ }^{2}$ Species groups are as proposed by Bennett et al. (2012).

${ }^{3}$ Genbank accession numbers for rplF sequences generated in this study are given in brackets.

${ }^{4} \mathrm{~N}$. mucosa NRL 9300 also displayed activity against each of these strains in the cross streak assay.

CS, cross streak assay; +, zone of inhibition present; -, zone of inhibition absent; no symbol, not done.

AO, agar overlay assay; values indicate average diameter of zone of inhibition ( $\mathrm{mm})$; -, zone of inhibition absent; no symbol, not done.

$n=3$ for each assay against each target. 


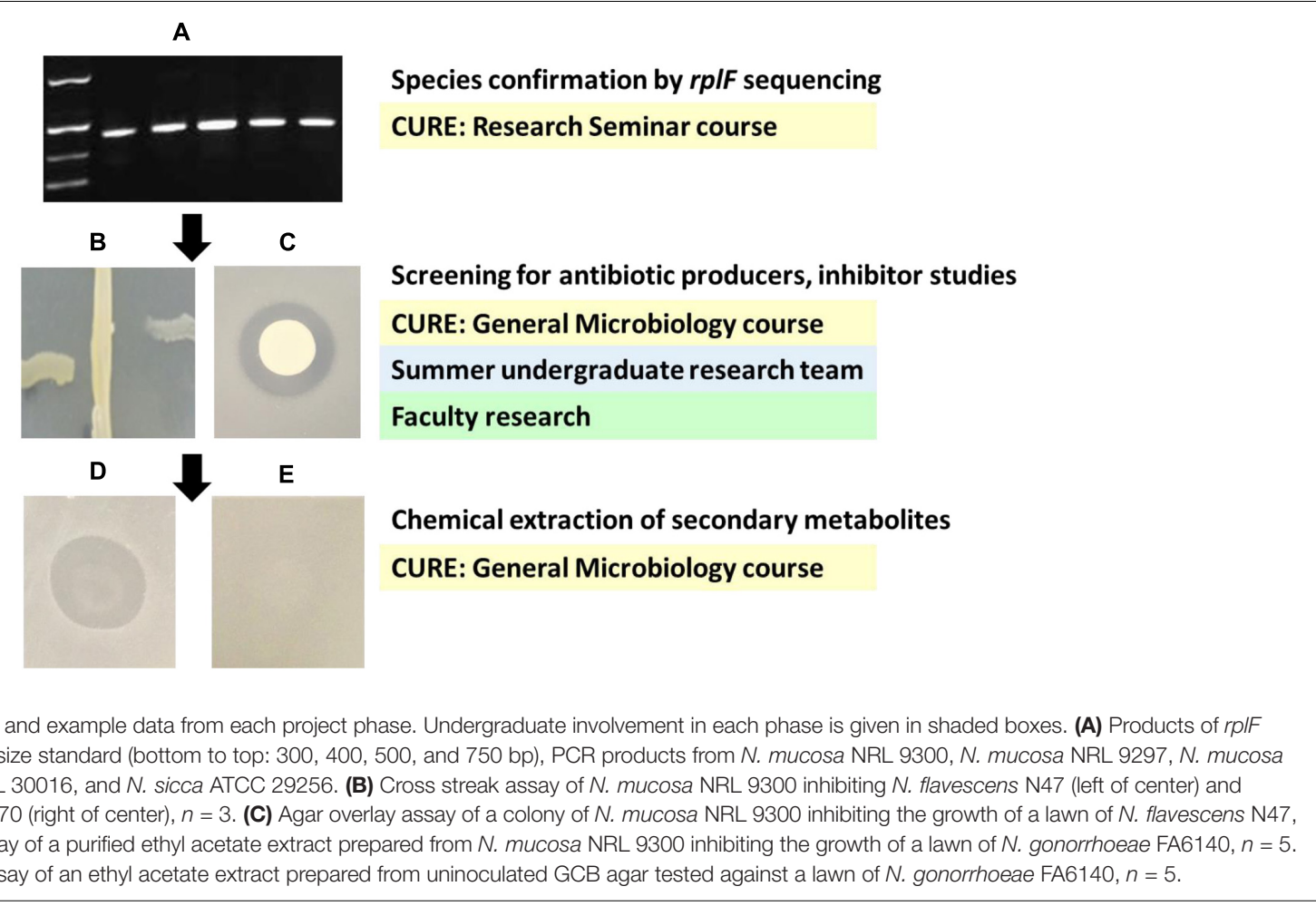

onto GCB agar. After $24 \mathrm{~h}$ of incubation, $10 \mathrm{ml}$ of melted GCB agar containing $10^{6} \mathrm{CFU}$ of a target strain was poured onto each plate and plates were reincubated. The diameter of the zone of inhibition surrounding the original producer strain was measured at $24 \mathrm{~h}(n=$ at least three trials per strain for each target). The number of CFU in the producer and target strain suspensions was confirmed by standard serial dilution and culture techniques.

\section{Inhibitor Studies and Chemical Extraction}

Inhibitor studies were used to investigate the antimicrobial activity displayed by N. mucosa NRL 9300 against $N$. gonorrhoeae strains MS11 and FA6140. Agar overlay assays in which the overlay contained 5, 50, or $500 \mathrm{U}$ bovine catalase (Worthington Biochemicals) per $\mathrm{ml}$ or $500 \mu \mathrm{g}$ bovine pancreatic trypsin (Sigma-Aldrich) per $\mathrm{ml}$ were performed to inhibit $\mathrm{H}_{2} \mathrm{O}_{2}$ (St. Amant et al., 2002) and protein (Jyssum and Allunans, 1982), respectively. Assays in which base and top agar contained $30 \mathrm{mM}$ HEPES buffer (Sigma Life Sciences) pH 7.4 were used to inhibit activity due to acidification (Knapp et al., 1975). All inhibitor assays were performed in triplicate and zones of inhibition were compared to control plates lacking inhibitors. We also tested for the possibility of DNA mediated antimicrobial activity in our agar overlay assay by testing N. mucosa NRL 9300 against a $\triangle$ comP mutant strain of $N$. gonorrhoeae MS11 that is incapable of DNA uptake. Controls for this assay included wild type strain $N$. gonorrhoeae MS11 and a complemented $\Delta$ comP mutant strain. All MS11 strains were generously provided by Dr. Magdalene So (Kim et al., 2019).
A crude extract containing the N. mucosa NRL 9300 antimicrobial compound was isolated using the technique to enrich for small, nonpolar secondary metabolites described by Hernandez et al. (2018). Briefly, a lawn of plate grown bacteria and their secreted substances were harvested by chopping the agar from a $100 \mathrm{~mm}$ diameter petri plate into approximately 1 $\mathrm{cm}^{2}$ pieces, freezing the material at $-70^{\circ} \mathrm{C}$, and performing an ethyl acetate extraction. The organic layer was dried, resuspended in methanol, and $30 \mu \mathrm{l}$ samples containing $300 \mu \mathrm{g}$ of extract, which represented approximately $20 \%$ of the yield, were spotted on an agar plate, allowed to dry, and tested against $N$. gonorrhoeae FA6140 using an agar overlay technique $(n=5)$. Control extractions were performed using uninoculated GBC agar (all chemicals were from Fisher Scientific).

\section{RESULTS}

\section{N. mucosa Isolates Secrete Antimicrobial Compounds}

We examined a panel of 36 nonpathogenic Neisseria isolates representing 15 species. The collection included 32 strains isolated from the human oropharynx, including reference strains utilized in the Human Microbiome Project (HMP) (Human Microbiome Jumpstart Reference Strains Consortium, 2010), and four strains from nonhuman animals. Although all strains were obtained from established isolate collections, the NRL strains are less well studied and their species identities had not been previously confirmed by genetic analyses. We used a validated technique to analyze a 413-bp region of the neisserial $r p l F$ gene, 
which encodes the $50 \mathrm{~S}$ ribosomal protein L6 (Bennett et al., 2014), from these strains and assigned isolates to neisserial species groups proposed by Bennett et al. (2012) (Table 1).

Delayed antagonism assays were used to test Neisseria species for their ability to produce antimicrobial compounds (Table 1, representative experiments shown in Figure 1). A semiquantitative cross-streak assay was used to screen all 36 isolates against three target strains: N. lactamica ATCC 23970, N. flavescens N47, and N. gonorrhoeae ATCC 43070. Five strains, all of which are members of the $N$. mucosa species group, displayed antimicrobial activity, although they did not exhibit identical activity profiles with respect to the target strains they inhibited (Table 1). Members of other neisserial species groups failed to show antineisserial activity under these conditions. Confirmatory agar overlay assays against the same three targets were carried out on all members of the $N$. mucosa group, yielding activity profiles identical to those seen in the initial screening experiments. Agar overlay zone of inhibition measurements suggest the $N$. mucosa producer strains may also differ from one another in their levels of antimicrobial activity, although these data should be interpreted with caution due to possible strain specific differences in growth rate and the limited quantitative power of this assay. The producer strain active against all screening targets, $N$. mucosa NRL 9300, was further tested against six additional N. gonorrhoeae strains, including the antibiotic resistant strain FA6140 (Veal et al., 2002), and displayed comparable activity against all gonococci in both assays (Table 1). Collectively, these data show nonpathogenic Neisseria species vary in their ability to secrete inhibitory compounds, and some $N$. mucosa strains are active against $N$. gonorrhoeae.

\section{N. mucosa NRL 9300 Activity Is Not Affected by Common Inhibitors and Can Be Isolated by Chemical Extraction}

Inhibitor studies were used to characterize the antimicrobial activity displayed by N. mucosa NRL 9300. Agar overlay assays against the target strains $N$. gonorrhoeae MS11 and FA6140 were performed in the presence of catalase, trypsin, and HEPES buffer. N. mucosa NRL 9300 displayed activity identical to that seen on control plates lacking inhibitor in all cases (Supplementary Table 1), suggesting the inhibitory activity is not due to $\mathrm{H}_{2} \mathrm{O}_{2}$, a trypsin sensitive protein toxin, or acidification. Kim et al. (2019) showed nonpathogenic Neisseria can kill N. gonorrhoeae in coculture assays via a mechanism in which $N$. gonorrhoeae takes up commensal DNA. These authors also demonstrated gonococcal DNA uptake mutants deficient in the protein ComP are no longer killed by this mechanism. We tested $N$. mucosa NRL 9300 inhibitory activity against a $\Delta \operatorname{comP}$ mutant strain of $N$. gonorrhoeae MS11 using our agar overlay assay. N. mucosa inhibited the mutant strain to the same degree that it inhibited wild type MS11 and a complemented $\triangle \operatorname{comP}$ mutant strain (Supplementary Table 1), indicating the inhibition detected by our delayed antagonism assays is not due to a DNA uptake mediated mechanism. Finally, an ethyl acetate extraction procedure was performed on plate-grown $N$. mucosa NRL
9300. The resulting crude extract inhibits $N$. gonorrhoeae strain FA6140 in an agar overlay assay (Figure 1), suggesting the antimicrobial activity displayed by $N$. mucosa may be due to a novel secondary metabolite.

\section{UNDERGRADUATE INVOLVEMENT AND TRANSFERRABLE STRATEGIES}

This project was designed to provide engaging undergraduate research experiences to as many students as possible while advancing the scientific objectives of the study. Although assessment of student learning outcomes was not a component of this work, a brief description of the project design and phasing from a pedagogical perspective may serve as a helpful model to others interested in designing human microbiome based antibiotic discovery experiences in undergraduate settings (Figure 1). The work described above involved over 70 students enrolled in three undergraduate biology classes in course-based undergraduate research experiences (CUREs) and provided a full-time summer research opportunity to a team of two students. Straightforward, relatively low-cost techniques were used throughout the project. Students involved in CUREs worked exclusively with nonpathogenic bacteria that could be handled safely in the teaching laboratory environment, while the faculty member and summer team carried out experiments utilizing $N$. gonorrhoeae under more stringent training and safety conditions. Participants in each research phase learned about the overarching goals of the neisserial antibiotic discovery project, and studied data generated by previous teams and relevant primary literature. Students in an upper-level genomics focused Research Seminar course carried out the DNA sequence based strain characterization in conjunction with learning about PubMLST and other databases, which they subsequently used to carry out additional independent bioinformatics projects. Participants in a General Microbiology course composed largely of students interested in health professions carried out initial screening of the isolate collection for inhibitory activity against the nonpathogenic target strains. This course represents students' first exposure to working with bacteria in the laboratory. Participation in this study provided students with opportunities to learn not only the assays used in the project, but also basic microbiology techniques such as media preparation, streak plating, and maintaining pure cultures, in the context of an engaging research question. In addition, the project complemented classroom activities addressing symbiosis, the human microbiome, and antibiotic resistance. The summer research team, which was composed of a first year student, a third year student, and the faculty member, replicated these assays, developed the agar overlay confirmatory assay, included screening against $N$. gonorrhoeae, and carried out inhibitor studies. Finally, N. mucosa chemical extractions were incorporated into a subsequent section of General Microbiology. Students presented their findings from various stages of the project to the broader campus community as part of a yearly Celebration of Student Scholarship, and the summer team also presented at regional and national conferences. This phased 
model blending faculty research interests, course goals, and both introductory and upper-level of undergraduate research involvement has allowed a broad range of students with various levels of experience to engage with and contribute to this project.

Our laboratory has a long history of Neisseria research, but this undergraduate antibiotic discovery model could easily be adapted to the study of other members of the human microbiome. The NIH Human Microbiome Project included both metagenomic analyses of body site samples from human volunteers and sequencing of approximately 800 previously isolated reference strains representing an array of species found in the microbiome (Human Microbiome Jumpstart Reference Strains Consortium, 2010; Human Microbiome Project Consortium, 2012). The HMP reference strains are well described in a searchable catalog ${ }^{2}$ and many are readily available from commercial strain collections. While the choice to screen HMP reference strains and/or other known strain collections for antibiotic production limits the number of representatives of the microbiome one can test, it has the advantage of avoiding the need for human subject research approval and safeguards that might be challenging in some undergraduate settings. Additionally, the ready availability of many reference strain genome sequences opens the possibility of linking functional screening to bioinformatics projects related to antibiotic discovery.

\section{DISCUSSION}

Bacteria residing in a common habitat display a wide array of competitive and cooperative interactions. The human oropharynx is home to over 700 prokaryotic species, including at least 12 Neisseria species, which are in intimate contact with one another, and with their host, in variety of niches (Liu et al., 2015). Several recent studies utilizing metagenomic data from the HMP have demonstrated $N$. mucosa is a common colonizer of the healthy oropharynx and displays niche specificity for sites including the buccal mucosa and supragingival plaque (Eren et al., 2014; Kraal et al., 2014; Mark Welch et al., 2014, 2016; Donati et al., 2016). For example, Kraal et al. (2014) reported supragingival plaque contained $N$. mucosa in 70/70 subjects tested, although the abundance of the organism varied among subjects. The normal mutualistic roles $N$. mucosa and other Neisseria species play as members of the healthy human microbiome are not well understood, however, we reasoned that nonpathogenic species may secrete secondary metabolites active against other Neisseria. Even though our study focused on a relatively small panel of isolates, we identified five $N$. mucosa group strains exhibiting antimicrobial activity against tester strains of nonpathogenic Neisseria, three of which also displayed activity against $N$. gonorrhoeae. This activity was retained in a crude ethyl acetate extract enriched for small nonpolar secondary metabolites.

The N. mucosa antigonococcal activity we observed does not appear to be due to previously described inhibitory mechanisms. Other genera residing in human microbiome, including vaginal

${ }^{2}$ https://www.hmpdacc.org/hmp/catalog/
Lactobacillus species, also display activity against $N$. gonorrhoeae in vitro. Lactobacillus-mediated inhibition is mediated by $\mathrm{H}_{2} \mathrm{O}_{2}$, bacteriocins, organic acids, or a combination of the three (St. Amant et al., 2002; Graver and Wade, 2011; Ruíz et al., 2015; Foschi et al., 2017). Inhibitors of these agents had no effect on $N$. mucosa antigonococcal activity. We also used DNA uptake mutants of $N$. gonorrhoeae to rule out the possibility we were detecting DNA mediated gonococcal killing previously described in nonpathogenic Neisseria (Kim et al., 2019). These findings suggest further characterization of the compounds secreted by N. mucosa NRL 9300 is warranted and raise the possibility nonpathogenic Neisseria may employ multiple mechanisms for inhibiting other bacteria. This would not be surprising given that each unique nonpathogenic species interacts with a variety of other organisms in a diverse array of dynamic body site habitats.

Natural products have not been well studied in the genus Neisseria as a whole, and to the best of our knowledge this is the first report describing secondary metabolite activity in $N$. mucosa. Future directions for this project include chemical and quantitative analyses of the components of the $N$. mucosa ethyl acetate extract, validation of antigonoccal activity in animal models, and examination of neisserial genomes for potential biosynthetic gene clusters. The antibiotic discovery potential we have identified in Neisseria species is likely also present in many other uncharacterized members of the human microbiome that await investigation. This demonstration project has shown undergraduate teams participating in CUREs and other guided research experiences can make continued contributions to this emerging area of natural products research.

\section{DATA AVAILABILITY STATEMENT}

The raw data supporting the conclusions of this article will be made available by the authors, without undue reservation.

\section{AUTHOR CONTRIBUTIONS}

EA, JO, and AF designed and conducted experiments and interpreted the data. EA developed the overall project, directed course-based research, and wrote the manuscript. All authors contributed to the article and approved the submitted version.

\section{FUNDING}

This work was supported by grants from the National Science Foundation (DUE-0969568) and the Concordia College Fuglestad-Torstveit Research Endowment.

\section{ACKNOWLEDGMENTS}

The authors thank members of the Concordia College General Microbiology and Research Seminar courses that contributed to this work, the Genomics Division of the Iowa Institute of Human Genetics for performing rplF sequencing, and Janne 
Cannon, Robert Nicholas, and Magdalene So for providing bacterial strains used in this study. This publication made use of the Neisseria Multi Locus Sequence Typing website sited at the University of Oxford. The development of this site has been funded by the Wellcome Trust and European Union.

\section{REFERENCES}

Bennett, J. S., Jolley, K. A., Earle, S. G., Corton, C., Bentley, S. D., Parkhill, J., et al. (2012). A genomic approach to bacterial taxonomy: an examination and proposed reclassification of species within the genus Neisseria. Microbiology 158, 1570-1580. doi: 10.1099/mic.0.056077-0

Bennett, J. S., Watkins, E. R., Jolley, K. A., Harrison, O. B., and Maiden, M. C. (2014). Identifying Neisseria species by use of the $50 \mathrm{~S}$ ribosomal protein L6 (rplF) gene. J. Clin. Microbiol. 52, 1375-1381. doi: 10.1128/JCM.03 529-13

Centers for Disease Control and Prevention (2012). Cephalosporin-Resistant Neisseria Gonorrhoeae Public Health Response Plan. Atlanta: CDC.

Custodio, R., Johnson, E., Liu, G., Tang, C. M., and Exley, R. M. (2020). Commensal Neisseria cinerea impairs Neisseria meningitidis microcolony development and reduces pathogen colonisation of epithelial cells. PLoS Pathog. 16:e1008372. doi: 10.1371/journal.ppat.1008372

Deasy, A. M., Guccione, E., Dale, A. P., Andrews, N., Evans, C. M., Bennett, J. S., et al. (2015). Nasal inoculation of the commensal Neisseria lactamica inhibits carriage of Neisseria meningitidis by young adults: a controlled human infection study. Clin. Infect. Dis. 60, 1512-1520. doi: 10.1093/cid/civ098

Donati, C., Zolfo, M., Albanese, D., Tin Truong, D., Asnicar, F., Iebba, V., et al. (2016). Uncovering oral Neisseria tropism and persistence using metagenomic sequencing. Nat. Microbiol. 1:16070. doi: 10.1038/nmicrobiol.2016.70

Donia, M. S., and Fischbach, M. A. (2015). Small molecules from the human microbiota. Science 349, 1254766. doi: 10.1126/science. 1254766

Dube, D. H. (2018). Design of a drug discovery course for non-science majors. Biochem. Mol. Biol. Educ. 46, 327-335. doi: 10.1002/bmb.21121

Eren, A. M., Borisy, G. G., Huse, S. M., and Mark Welch, J. L. (2014). Oligotyping analysis of the human oral microbiome. Proc. Natl. Acad. Sci. U.S.A. 111, E2875-E2884. doi: 10.1073/pnas.1409644111

European Centre for Disease Prevention and Control (2019). Response Plan to Control and Manage The Threat of Multi- and Extensively Drug-Resistant Gonorrhoeae in Europe - 2019 Update. Stockholm: ECDC.

Eyre, D. W., Sanderson, N. D., Lord, E., Regisford-Reimmer, N., Chau, K., Barker, L., et al. (2018). Gonorrhea treatment failure caused by a Neisseria gonorrhoeae strain with combined ceftriaxone and high-level azithromycin resistance, England, February 2018. Euro Surveill. 23:1800323. doi: 10.2807/ 1560-7917.ES.2018.23.27.1800323

Foschi, C., Salvo, M., Cevenini, R., Parolin, C., Vitali, B., and Marangoni, A. (2017). Vaginal lactobacilli reduce Neisseria gonorrhoeae viability through multiple strategies: an in vitro study. Front. Cell. Infect. Microbiol. 7:502. doi: 10.3389/ fcimb.2017.00502

Genné-Bacon, E. A., and Bascom-Slack, C. A. (2018). The PARE Project: a short course-based research project for national surveillance of antibiotic-resistant microbes in environmental samples. J. Microbiol. Biol. Educ. 19, 19-97. doi: 10.1128/jmbe.v19i3.1603

Graver, M. A., and Wade, J. J. (2011). The role of acidification in the inhibition of Neisseria gonorrhoeae by vaginal lactobacilli during anaerobic growth. Ann. Clin. Microbiol. Antimicrob. 10:8. doi: 10.1186/1476-0711-10-8

Hernandez, S., Tsang, T., Bascom-Slack, C., Broderick, N., and Handelsman, J. (2018). Tiny Earth: A Research Guide to Studentsourcing Antibiotic Discovery. Acton, MA: XanEdu Press.

Hoskisson, P. A., Aldridge, P., and Bowater, L. (2015). Inspiring STEM undergraduates to tackle the AMR crisis. FEMS Microbiol. Lett. 362:fnv138. doi: 10.1093/femsle/fnv138

Human Microbiome Jumpstart Reference Strains Consortium, Nelson, K. E., Weinstock, G. M., Highlander, S. K., Worley, K. C., Creasy, H. H., et al. (2010). A catalog of reference genomes from the human microbiome. Science 328, 994-999. doi: 10.1126/science. 1183605

\section{SUPPLEMENTARY MATERIAL}

The Supplementary Material for this article can be found online at: https://www.frontiersin.org/articles/10.3389/fmicb. 2020.577762/full\#supplementary-material

Human Microbiome Project Consortium (2012). A framework for human microbiome research. Nature 486, 215-221. doi: 10.1038/nature11209

Jolley, K. A., Bray, J. E., and Maiden, M. (2018). Open-access bacterial population genomics: BIGSdb software, the PubMLST.org website and their applications. Wellcome Open Res. 3:124. doi: 10.12688/wellcomeopenres.14826.1

Jyssum, K., and Allunans, J. (1982). Three types of growth inhibition among strains of Neisseria meningitidis isolated from patients in Norway. Acta Pathol. Microbiol. Immunol. Scand. B 90, 335-340. doi: 10.1111/j.1699-0463.1982. tb00128.x

Kellogg, D. S., Peacock, W. L., Deacon, W. E., Brown, L., and Pirkle, D. I. (1963). Neisseria gonorrhoeae. I. Virulence genetically linked to clonal variation. J. Bacteriol. 85, 1274-1279.

Kim, W. J., Higashi, D., Goytia, M., Rendón, M. A., Pilligua-Lucas, M., Bronnimann, M., et al. (2019). Commensal Neisseria kill Neisseria gonorrhoeae through a DNA-dependent mechanism. Cell Host Microbe 26, 228-239. doi: 10.1016/j.chom.2019.07.003

Knapp, J. S., Falkow, S., and Holmes, K. K. (1975). Reevaluation of bacteriocinogeny in Neisseria gonorrhoeae. J. Clin. Pathol. 28, 274-278. doi: 10.1136/jcp.28.4.274

Kraal, L., Abubuker, S., Kota, K., Fischbach, M. A., and Mitreva, M. (2014). The prevalence of species and strains in the human microbiome: a resource for experimental efforts. PLoS One 9:e97279. doi: 10.1371/journal.pone.0097279

Liu, G., Tang, C. M., and Exley, R. M. (2015). Non-pathogenic Neisseria: members of an abundant, multi-habitat, diverse genus. Microbiology 161, 1297-1312. doi: $10.1099 / \mathrm{mic} .0 .000086$

Maiden, M. C. (1998). Horizontal genetic exchange, evolution, and spread of antibiotic resistance in bacteria. Clin. Infect. Dis. 27(Suppl. 1), S12-S20. doi: $10.1086 / 514917$

Mark Welch, J. L., Rossetti, B. J., Rieken, C. W., Dewhirst, F. E., and Borisy, G. G. (2016). Biogeography of a human oral microbiome at the micron scale. Proc. Natl. Acad. Sci. U.S.A. 113, E791-E800. doi: 10.1073/pnas.1522149113

Mark Welch, J. L., Utter, D. R., Rossetti, B. J., Mark Welch, D. B., Eren, A. M., and Borisy, G. G. (2014). Dynamics of tongue microbial communities with singlenucleotide resolution using oligotyping. Front. Microbiol. 5:568. doi: 10.3389/ fmicb.2014.00568

Marri, P. R., Paniscus, M., Weyand, N. J., Rendón, M. A., Calton, C. M., Hernández, D. R., et al. (2010). Genome sequencing reveals widespread virulence gene exchange among human Neisseria species. PLoS One 5:e11835. doi: 10.1371/ journal.pone.0011835

Milshteyn, A., Colosimo, D. A., and Brady, S. F. (2018). Accessing bioactive natural products from the human microbiome. Cell Host Microbe 23, 725-736. doi: 10.1016/j.chom.2018.05.013

Rowley, J., Vander Hoorn, S., Korenromp, E., Low, N., Unemo, M., Abu-Raddad, L. J., et al. (2019). Chlamydia, gonorrhea, trichomoniasis and syphilis: global prevalence and incidence estimates, 2016. Bull. World Health Organ. 97, 548562. doi: 10.2471/BLT.18.228486

Ruíz, F. O., Pascual, L., Giordano, W., and Barberis, L. (2015). Bacteriocins and other bioactive substances of probiotic lactobacilli as biological weapons against Neisseria gonorrhoeae. Pathog. Dis. 73:ftv013. doi: 10.1093/femspd/ftv013

Smyth, D. S. (2017). An authentic course-based research experience in antibiotic resistance and microbial genomics. Sci. Educ. Civic Engag. 9, 59-65.

Spratt, B. G., Bowler, L. D., Zhang, Q. Y., Zhou, J., and Smith, J. M. (1992). Role of interspecies transfer of chromosomal genes in the evolution of penicillin resistance in pathogenic and commensal Neisseria species. J. Mol. Evol. 34, 115-125. doi: 10.1007/BF00182388

St. Amant, D. C., Valentin-Bon, I. E., and Jerse, A. E. (2002). Inhibition of Neisseria gonorrhoeae by Lactobacillus species that are commonly isolated from the female genital tract. Infect. Immun. 70, 7169-7171. doi: 10.1128/iai.70.12.71697171.2002 
Unemo, M., Seifert, H. S., Hook, E. W. III, Hawkes, S., Ndowa, F., and Dillon, J. R. (2019). Gonorrhoea. Nat. Rev. Dis. Primers 5:79. doi: 10.1038/s41572-0190128-6

Veal, W. L., Nicholas, R. A., and Shafer, W. M. (2002). Overexpression of the MtrC-MtrD-MtrE efflux pump due to an $m t r R$ mutation is required for chromosomally mediated penicillin resistance in Neisseria gonorrhoeae. J. Bacteriol. 184, 5619-5624. doi: 10.1128/jb.184.20.5619-5624. 2002

Weyand, N. J. (2017). Neisseria models of infection and persistence in the upper respiratory tract. Pathog. Dis. 75:ftx031. doi: 10.1093/femspd/ftx031

Whiley, D. M., Jennison, A., Pearson, J., and Lahra, M. M. (2018). Genetic characterisation of Neisseria gonorrhoeae resistant to both ceftriaxone and azithromycin. Lancet Infect. Dis. 18, 717-718. doi: 10.1016/S1473-3099(18) 30340-2

Wilson, M. R., Zha, L., and Balskus, E. P. (2017). Natural product discovery from the human microbiome. J. Biol. Chem. 292, 8546-8552. doi: 10.1074/jbc.R116. 762906
World Health Organization, Department of Reproductive Health and Research (2012). Global Action Plan to Control the Spread and Impact of Antimicrobial Resistance in Neisseria gonorrhoeae. Geneva: WHO.

Zipperer, A., Konnerth, M. C., Laux, C., Berscheid, A., Janek, D., Weidenmaier, C., et al. (2016). Human commensals producing a novel antibiotic impair pathogen colonization. Nature 535, 511-516. doi: 10.1038/nature18634

Conflict of Interest: The authors declare that the research was conducted in the absence of any commercial or financial relationships that could be construed as a potential conflict of interest.

Copyright $\odot 2020$ Aho, Ogle and Finck. This is an open-access article distributed under the terms of the Creative Commons Attribution License (CC BY). The use, distribution or reproduction in other forums is permitted, provided the original author(s) and the copyright owner(s) are credited and that the original publication in this journal is cited, in accordance with accepted academic practice. No use, distribution or reproduction is permitted which does not comply with these terms. 